\title{
Betti Numbers of Weighted Oriented Graphs
}

\author{
Beata Casiday \\ Department of Mathematics \\ Yale University \\ New Haven, U.S.A. \\ bea.r.casiday@gmail.com
}

\author{
Selvi Kara \\ Department of Mathematics and Statistic \\ University of South Alabama \\ Mobile, U.S.A. \\ selvi@southalabama.edu
}

Submitted: Sep 23, 2020; Accepted: May 21, 2021; Published: Jun 4, 2021

(C) The authors. Released under the CC BY-ND license (International 4.0).

\begin{abstract}
Let $\mathcal{D}$ be a weighted oriented graph and $I(\mathcal{D})$ be its edge ideal. In this paper, we investigate the Betti numbers of $I(\mathcal{D})$ via upper-Koszul simplicial complexes, Betti splittings and the mapping cone construction. In particular, we provide recursive formulas for the Betti numbers of edge ideals of several classes of weighted oriented graphs. We also identify classes of weighted oriented graphs whose edge ideals have a unique extremal Betti number which allows us to compute the regularity and projective dimension for the identified classes. Furthermore, we characterize the structure of a weighted oriented graph $\mathcal{D}$ on $n$ vertices such that $\operatorname{pdim}(R / I(\mathcal{D}))=n$ where $R=k\left[x_{1}, \ldots, x_{n}\right]$.
\end{abstract}

Mathematics Subject Classifications: 05C25, 05C75, 13D02, 13F20

\section{Introduction}

An oriented graph is an ordered pair $\mathcal{D}=(V(\mathcal{D}), E(\mathcal{D}))$ with the vertex set $V(\mathcal{D})$, the edge set $E(\mathcal{D})$ and an underlying graph $G$ on which each edge is given an orientation. If $e=\{x, y\}$ is an edge in $G$ and $e$ is oriented from $x$ to $y$ in $\mathcal{D}$, we denote the oriented edge by $(x, y)$ to reflect the orientation. In contrast to directed graphs, multiple edges or loops are not allowed in oriented graphs. An oriented graph $\mathcal{D}$ is called vertex-weighted oriented (or simply weighted) if each vertex is assigned a weight by a function $w: V(\mathcal{D}) \rightarrow \mathbb{N}^{+}$ called a weight function. For simplicity, we set $w_{i}=w\left(x_{i}\right)$ for each $x_{i} \in V(\mathcal{D})$. If the weight value $w_{i}$ of vertex $x_{i}$ is one, we say $x_{i}$ has a simple weight in $\mathcal{D}$. Otherwise, we say $x_{i}$ has a non-simple weight in $\mathcal{D}$.

Let $\mathcal{D}$ be a weighted oriented graph with the vertex set $V(\mathcal{D})=\left\{x_{1}, \ldots, x_{n}\right\}$ and $R=k\left[x_{1}, \ldots, x_{n}\right]$ be a polynomial ring over a field $k$. By identifying vertices of $\mathcal{D}$ with variables in $R$, the edge ideal of a weighted oriented graph $\mathcal{D}$ is defined as

$$
I(\mathcal{D})=\left(x_{i} x_{j}^{w_{j}}:\left(x_{i}, x_{j}\right) \in E(\mathcal{D})\right) .
$$


If all vertices of $\mathcal{D}$ have simple weights, then $I(\mathcal{D})$ is the edge ideal of the (undirected, unweighted) underlying graph of $\mathcal{D}$. Edge ideal of undirected, unweighted graphs are studied extensively in the literature (see [2]). The minimal generators of $I(\mathcal{D})$ display only the weights of target vertices for each edge. If a vertex has only outgoing edges from it, we call it a source vertex. Since weights of source vertices do not appear in the minimal generators of $I(\mathcal{D})$, we shall assume that $w_{i}=1$ if $x_{i}$ is a source vertex.

One of the known appearances of edge ideals of weighted oriented graphs is in the algebraic coding theory literature (see $[8,16])$. In particular, the ideal $I=\left(x_{i} x_{j}^{w_{j}}: 1 \leqslant\right.$ $i<j \leqslant n$ ) where $2 \leqslant w_{1} \leqslant \cdots \leqslant w_{n}$ is the initial ideal of the vanishing ideal $I(\mathcal{X})$ of a projective nested cartesian set $\mathcal{X}$ with respect to the lexicographic order (see [8, Proposition 2.11]). Projective nested cartesian codes are images of degree $d$ evaluation maps on $\mathcal{X}$ and these type of evaluation codes are introduced to generalize the classical projective Reed-Muller type codes. Note that the ideal $I$ is the edge ideal of a weighted naturally oriented complete graph on $n$ vertices with the edge set $\left\{\left(x_{i}, x_{j}\right): 1 \leqslant i<\right.$ $j \leqslant n\}$ such that $x_{1}$ is a source vertex and all other vertices are of non-simple weight. Algebraic invariants of $I$ is used in detecting "good" projective nested cartesian codes, for instance, the Castelnuovo-Mumford regularity of $R / I$, denoted by $\operatorname{reg}(R / I)$, is a strict upper bound for an "optimal" degree $d$.

Edge ideals of weighted oriented graphs are fairly new objects in the combinatorial commutative algebra community and there have been a few papers investigating algebraic properties and invariants of these objects (see $[5,12,14,18,19])$. There has been an extensive literature on the edge ideals of (unweighted, unoriented) graphs, and one of the reasons for such fruitful outcomes is due to the squarefree nature of edge ideals of graphs. On the other hand, edge ideals of weighted oriented graphs are not squarefree in general, so many of the established squarefree connections such as Hochster's formula and independence complexes are no longer available in studying our objects of interest. Furthermore, descriptions of edge ideals of weighted oriented graphs differ depending on the orientation and positions of non-simple weights, making it more complicated to obtain general results for this class of ideals.

Our general goal is to address these issues in the study of Betti numbers of edge ideals of weighted oriented graphs. One of the essential elements of the paper is the upperKoszul simplicial complex. The following Hochster-like formula given in [3, Theorem 2.2] exploits the structure of upper Koszul-simplicial complexes and allows one to compute Betti numbers of $I(\mathcal{D})$ in terms of the holes of upper-Koszul simplicial complexes.

$$
\beta_{i, \mathbf{b}}(I)=\operatorname{dim}_{k} \widetilde{H}_{i-1}\left(K_{\mathbf{b}}(I) ; k\right)
$$

where $K_{\mathbf{b}}(I)$ is the upper-Koszul simplicial complex of a monomial ideal $I$ at multidegree $\mathbf{b} \in \mathbb{N}^{n}$.

Current literature on algebraic invariants of edge ideals of weighted oriented graphs is obtained by focusing on specific classes of graphs with a predetermined orientation or weight assumptions. For instance, in [19], authors investigate the regularity and the projective dimension of edge ideals of weighted cycles and rooted forests with the assumption that all non-source vertices are of non-simple weight and graphs are naturally oriented (i.e., all edges are oriented in the same direction). A more general case, class of weighted naturally oriented paths and cycles under any weight distribution is studied in [5]. All the formulas provided in $[5,19]$ heavily depend on the orientation and weight 
distributions. In order to obtain more general results, we investigate the Betti numbers and provide recursive formulas for these invariants. With this approach, we successfully recover several results from [19] and suggest an explanation for the nature of regularity formulas given in [5].

If $\mathcal{D}$ is a weighted oriented graph on $n$ vertices, then the projective dimension of $R / I(\mathcal{D})$ is at most $n$ by Hilbert's Syzygy Theorem. One of the main results of the paper classifies all weighted oriented graphs such that the upper bound for the projective dimension is tight.

Theorem 1 (Theorem 19). Let $\mathcal{D}$ be a weighted oriented graph on the vertices $V(\mathcal{D})=$ $\left\{x_{1}, \ldots, x_{n}\right\}$. Then

$$
\operatorname{pdim}(R / I(\mathcal{D}))=n
$$

if and only if there is an edge $e=\left(x_{j}, x_{i}\right)$ oriented towards $x_{i}$ for each $x_{i} \in V(\mathcal{D})$ such that $x_{j}$ has a non-simple weight. Furthermore,

$$
\operatorname{pdim}(R / I(\mathcal{D}))=n \text { if and only if } \beta_{n, b}(R / I(\mathcal{D})) \neq 0
$$

where $\boldsymbol{b}=\left(w_{1}, \ldots, w_{n}\right) \in \mathbb{N}^{n}$.

Note that a weighted naturally oriented cycle belongs to the class of weighted oriented graphs described in Theorem 19 if none of its vertices is of simple weight. As a result, we recover [19, Theorem 1.4.].

We achieve Theorem 19 by concluding that edge ideals of weighted oriented graphs with maximum projective dimension have a unique extremal Betti number and it occurs at the multidegree $\mathbf{b}=\left(w_{1}, \ldots, w_{n}\right) \in \mathbb{N}^{n}$. In addition, we show that $\beta_{n-1, \mathbf{b}}$ is the unique extremal Betti number for edge ideals of classes of weighted oriented complete graphs and weighted rooted graphs on $n$ vertices where $\mathbf{b}=\left(w_{1}, \ldots, w_{n}\right) \in \mathbb{N}^{n}$. As a result, we provide the following formulas for the regularity and projective dimension.

Theorem 2 (Theorem 25 and Theorem 28). Let $\mathcal{D}$ be a weighted oriented graph on the vertices $\left\{x_{1}, \ldots, x_{n}\right\}$.

(a) If $\mathcal{D}$ is a weighted rooted graph with the root vertex $x_{1}$ and $w_{i} \geqslant 2$ for all $i \neq 1$, then

$$
\operatorname{pdim}(R / I(\mathcal{D}))=n-1 \text { and } \operatorname{reg}(R / I(\mathcal{D}))=\sum_{i=1}^{n} w_{i}-n+1
$$

(b) If $\mathcal{D}$ is a naturally oriented weighted complete graph such that $w_{p}>1$ for some $p \geqslant 2$, then

$$
\operatorname{pdim}(R / I(\mathcal{D}))=n-1 \text { and } \operatorname{reg}(R / I(\mathcal{D}))=\sum_{i=1}^{n} w_{i}-n+1
$$

As an immediate consequence of Theorem 25, one can obtain the regularity and projective dimension of a weighted rooted forest with non-simple weights, recovering [19, Theorem 1.2. and Theorem 1.3.]. The edge ideal $I(\mathcal{D})$ in Theorem 28 is the initial ideal of the vanishing ideal of a projective nested cartesian set and $\operatorname{reg}(R / I(\mathcal{D}))$ is a strict 
upper bound for the optimal degree of the evaluation map used in creating projective nested cartesian codes.

In addition, we provide recursive formulas for the Betti numbers of edge ideals of weighted oriented graphs where the first one is obtained via Betti splittings and the second one is obtained by employing the mapping cone construction.

Theorem 3 (Theorem 30). Let $\mathcal{D}_{n}$ denote a weighted oriented complete graph on $n$ vertices $\left\{x_{1}, \ldots, x_{n}\right\}$. Suppose $\mathcal{D}_{n}$ has at least one sink vertex. Then, for all $i>1$,

$$
\beta_{i, j}\left(R / I\left(\mathcal{D}_{n}\right)\right)= \begin{cases}\beta_{i, j}\left(R / I\left(\mathcal{D}_{n-1}\right)\right)+\left(\begin{array}{c}
n-1 \\
i
\end{array}\right)+\beta_{i-1, j-w_{n}}\left(R / I\left(\mathcal{D}_{n-1}\right)\right) & : j=i+w_{n} \\
\beta_{i, j}\left(R / I\left(\mathcal{D}_{n-1}\right)\right)+\beta_{i-1, j-w_{n}}\left(R / I\left(\mathcal{D}_{n-1}\right)\right) & : j \neq i+w_{n} .\end{cases}
$$

The following result is a natural extension of $[6,2.1 .1$. Theorem $]$ and $[7,2.7$. Theorem].

Theorem 4 (Theorem 33). Let $\mathcal{D}$ be a weighted oriented graph on the vertices $\left\{x_{1}, \ldots, x_{n}\right\}$ with a leaf $x_{n}$. Suppose $x_{n}$ is a sink vertex. Then, for any $i$ and $j$, we have

$$
\left.\beta_{i, j}(R / I(\mathcal{D}))=\beta_{i, j}\left(R / I\left(\mathcal{D} \backslash x_{n}\right)\right)+\beta_{i-1, j-w-1}\left(R / I\left(\mathcal{D} \backslash x_{n}\right): x_{n-1}\right)\right) .
$$

As an application of Theorem 33, we discuss the nature of the regularity formulas given in [5] for weighted naturally oriented paths.

Our paper is organized as follows. In Section 2, we recall the necessary terminology and results which will be used in the paper. In Section 3, we provide general results by relating algebraic invariants of edge ideals of weighted oriented graphs and their induced weighted oriented subgraphs. We also show that reducing weight of a non-simple sink vertex (i.e., a vertex with only incoming edges towards it) keeps projective dimension unchanged (Corollary 17). In Section 4, we prove Theorem 19 and Theorem 25. Section 5 is devoted to weighted oriented complete graphs on $n$ vertices. In particular, we provide formulas for the regularity and projective dimension when the edges are oriented in the "natural" way, i.e., $\left(x_{i}, x_{j}\right)$ for any $1 \leqslant i<j \leqslant n$. We also prove Theorem 30 in this section. In Section 6, we use the mapping cone construction and obtain recursive formulas for Betti numbers of weighted oriented graphs in Theorem 33. Finally, in Section 7, we raise some questions about the behavior of Betti numbers of edge ideals under weight reduction process.

\section{Preliminaries}

In this section, we collect the notation and terminology that will be used throughout the paper.

Let $R=k\left[x_{1}, \ldots, x_{n}\right]$ be a polynomial ring over a field $k$ and $M$ be a finitely generated $R$ module. Then the minimal free resolution of $M$ over $R$ is of the form

$$
0 \longrightarrow \bigoplus_{j \in \mathbb{Z}} R(-j)^{\beta_{p, j}(M)} \longrightarrow \bigoplus_{j \in \mathbb{Z}} R(-j)^{\beta_{p-1, j}(M)} \longrightarrow \cdots \longrightarrow \bigoplus_{j \in \mathbb{Z}} R(-j)^{\beta_{0, j}(M)} \longrightarrow M \longrightarrow 0 .
$$

The exponents $\beta_{i, j}(M)$ are invariants of the module called the Betti numbers of $M$ and these invariants encode all the information about the minimal free resolution of a module. In general, 
it is difficult to explicitly compute the Betti numbers. A common approach to go around this issue is to investigate coarser invariants of the module associated to Betti numbers. In this paper, we focus on the Castelnuovo-Mumford regularity (or simply, regularity), projective dimension and the extremal Betti numbers of $M=R / I$ where $I$ is a homogeneous ideal of $R$. The Castelnuovo-Mumford regularity and the projective dimension of $R / I$ are defined as

$$
\operatorname{reg}(R / I)=\max \left\{j-i: \beta_{i, j}(R / I) \neq 0\right\}
$$

and

$$
\operatorname{pdim}(R / I)=\max \left\{i: \beta_{i, j}(R / I) \neq 0\right\} .
$$

A Betti number $\beta_{k, l}(R / I) \neq 0$ is called extremal if $\beta_{i, j}(R / I)=0$ for all pairs $(i, j) \neq(k, l)$ with $i \geqslant k$ and $j \geqslant l$. In other words, extremal Betti numbers occupy the upper left corner of a block of zeroes in the Betti diagram of $R / I$ in Macaulay 2. The notion of extremal Betti numbers are introduced in [3] as a refinement of the notion of the regularity and one can read off the regularity and projective dimension from the extremal Betti numbers. Particularly, $R / I$ has the unique extremal Betti number if and only if $\beta_{p, p+r}(R / I) \neq 0$ where $p=\operatorname{pdim}(R / I)$ and $r=\operatorname{reg}(R / I)$.

\subsection{Upper-Koszul Simplicial Complexes}

An important connection in the field of combinatorial commutative algebra is the StanleyReisner correspondence which allows one to relate a squarefree monomial ideal with a simplicial complex (see [11]). As part of the Stanley-Reisner theory, one can compute the Betti numbers of a squarefree monomial ideal through dimensions of holes of a simplicial complex through the Hochster's formula. When the ideal $I$ is no longer squarefree, there is no Stanley-Reisner complex associated to $I$ and Hochster's formula cannot be applied directly. However, Bayer, Charalambous, and Popescu introduced the upper Koszul simplicial complex in [3] and provided a Hochster-like formula to compute the multigraded Betti numbers of any monomial ideal.

Definition 5. Let $I$ be a monomial ideal in $R=k\left[x_{1}, \ldots, x_{n}\right]$. The upper-Koszul simplicial complex of $I$ at multidegree $\mathbf{b}=\left(b_{1}, \ldots, b_{n}\right) \in \mathbb{N}^{n}$ is

$$
K_{\mathbf{b}}(I)=\left\{F \subseteq\left\{x_{1}, \ldots, x_{n}\right\}: \frac{x^{\mathbf{b}}}{x^{F}} \in I\right\}
$$

where $x^{\mathbf{b}}:=\prod_{i=1}^{n} x_{i}^{b_{i}}$ and $x^{F}:=\prod_{x_{i} \in F} x_{i}$.

Theorem 6. [3][Theorem 2.2] Given a monomial ideal $I$ in $R=k\left[x_{1}, \ldots, x_{n}\right]$, the multigraded Betti numbers of I are

$$
\beta_{i, \boldsymbol{b}}(I)=\operatorname{dim}_{k} \widetilde{H}_{i-1}\left(K_{\boldsymbol{b}}(I) ; k\right)
$$

where $\boldsymbol{b} \in \mathbb{N}^{n}$.

Remark 7. Let $I$ be monomial ideal in $R=k\left[x_{1}, \ldots, x_{n}\right]$ and $\mathbf{b} \in \mathbb{N}^{n}$. If $x^{\mathbf{b}}$ is not equal to a least common multiple of some of the minimal generators of $I$, then $K_{\mathbf{b}}(I)$ is a cone over some subcomplex. Therefore, all non-zero Betti numbers of $I$ occurs in $\mathbb{N}^{n}$-graded degrees $\mathbf{b}$ such that $x^{\mathbf{b}}$ is equal to a least common multiple of some minimal generators of $I$. 


\subsection{Betti Splitting}

In the study of Betti numbers of a monomial ideal $I$, one natural approach is to break down the ideal $I$ into smaller pieces and express the Betti numbers of $I$ in terms of the Betti numbers of the smaller pieces. This strategy was first introduced by Eliahou and Kervaire in [9] for monomial ideals and studied in more detail by Francisco, Hà, and Van Tuyl in [10].

Definition 8. Let $I, J$, and $K$ be monomial ideals with generating sets $\mathcal{G}(I), \mathcal{G}(J)$, and $\mathcal{G}(K)$ such that $\mathcal{G}(I)$ is the disjoint union of $\mathcal{G}(J)$ and $\mathcal{G}(K)$. Then $I=J+K$ is called a Betti splitting if

$$
\beta_{i, j}(R / I)=\beta_{i, j}(R / J)+\beta_{i, j}(R / K)+\beta_{i-1, j}(R / J \cap K)
$$

for all $i, j>1$.

The following result is quite useful as it provides a sufficient condition for $I=J+K$ to be a Betti splitting by considering just the generators of $I$.

Theorem 9. [10][Corollary 2.7] Suppose that $I=J+K$ where $\mathcal{G}(J)$ contains all the generators of $I$ divisible by some variables $x_{i}$ and $\mathcal{G}(K)$ is a non-empty set containing the remaining generators of $I$. If $J$ has a linear resolution, then $I=J+K$ is a Betti splitting.

\section{General Results}

In this section, we recall the notion of inducedness for weighted oriented graphs and introduce a new process called weight reduction process of a weighted oriented graph. Weight reductions of a weighted oriented graph $\mathcal{D}$ have the same underlying graph as $\mathcal{D}$ and the weight of one of the vertices of non-simple weight in $\mathcal{D}$ is reduced by one. In the main result of this section, we show that the weight reduction of a non-trivial sink vertex keeps the projective dimension unchanged.

Definition 10. Let $\mathcal{D}$ be a weighted oriented graph with the underlying graph $G$. We say $\mathcal{D}^{\prime}$ is an induced weighted oriented subgraph of $\mathcal{D}$ if

- the underlying graph of $\mathcal{D}^{\prime}$ is an induced subgraph of $G$,

- orientation of $\mathcal{D}^{\prime}$ is induced from $\mathcal{D}$, i.e., $E\left(\mathcal{D}^{\prime}\right) \subseteq E(\mathcal{D})$, and

- $w_{x}\left(\mathcal{D}^{\prime}\right)=w_{x}(\mathcal{D})$ for all $x \in V\left(\mathcal{D}^{\prime}\right)$

where $w_{x}(\mathcal{D})$ denotes the weight of $x$ in $\mathcal{D}$ and $w_{x}\left(\mathcal{D}^{\prime}\right)$ denotes the weight of $x$ in $\mathcal{D}^{\prime}$. We shall use this notation throughout the text while considering weight of a vertex in two different weighted oriented graphs.

Let $\mathcal{D}$ be a weighted oriented graph and $x$ be a vertex in $\mathcal{D}$. We use $\mathcal{D} \backslash x$ to denote the induced weighted oriented graph of $\mathcal{D}$ which is obtained from $\mathcal{D}$ by deleting the vertex $x$ and all oriented edges incident to $x$ in $\mathcal{D}$.

It is well-known that Betti numbers of edge ideals of induced subgraphs for unweighted unoriented graphs can not exceed that of the original graph (see [4, Lemma 4.2]). We provide an analog of this well-known result for weighted oriented graphs in the following lemma.

Lemma 11. Let $\mathcal{D}^{\prime}$ be an induced weighted oriented subgraph of $\mathcal{D}$. Then, for all $i, j \geqslant 0$, we have

$$
\beta_{i, j}\left(I\left(\mathcal{D}^{\prime}\right)\right) \leqslant \beta_{i, j}(I(\mathcal{D}))
$$


Proof. Since the notion of inducedness for weighted oriented graphs is an extension of inducedness for unweighted, unoriented graphs, we can adopt the proof of [4, Lemma 4.2] to obtain the inequality.

Corollary 12. Let $\mathcal{D}^{\prime}$ be an induced weighted oriented subgraph of $\mathcal{D}$. Then

$$
\operatorname{pdim}\left(R / I\left(\mathcal{D}^{\prime}\right)\right) \leqslant \operatorname{pdim}(R / I(\mathcal{D})) \text { and } \operatorname{reg}\left(R / I\left(\mathcal{D}^{\prime}\right)\right) \leqslant \operatorname{reg}(R / I(\mathcal{D})) .
$$

In the study of edge ideals of weighted oriented graphs, we assume that source vertices have simple weights. In a similar vein, one can ask whether the same treatment can be applied to sink vertices. In [14], authors assume that sinks vertices have simple weights along with source vertices while investigating Cohen-Macaulayness of edge ideals. In the investigation of algebraic invariants such as regularity and projective dimension, reducing the weight of a sink vertex of non-simple weight may effect these algebraic invariants. Thus, one needs to carefully consider the effects of weight reduction process on the multigraded Betti numbers of edge ideals and its invariants associated to Betti numbers. For this purpose, we introduce the explicit definition of the weight reduction process.

Definition 13. Let $\mathcal{D}$ be a weighted oriented graph. We obtain new weighted oriented graphs from $\mathcal{D}$ through a process called weight reduction process such that it

- preserves the vertices and oriented edges of $\mathcal{D}$,

- preserves weights of all vertices of $\mathcal{D}$ other than one vertex of non-simple weight, say $x$, and reduces the weight of $x$ by one.

A weighted oriented graph obtained from $\mathcal{D}$ through a weight reduction process is called a weight reduction of $\mathcal{D}$. If all vertices of $\mathcal{D}$ are of simple weight, it has no weight reductions.

We use the following notation throughout this section.

Note 14 . Let $\mathcal{D}$ be a weighted oriented graph on $n$ vertices. Suppose $\mathcal{D}$ has at least one sink vertex of non-simple weight, say $x_{p}$, such that $w_{p}(\mathcal{D})=w>1$. Then in-neighbors of $x_{p}$, denoted by $N_{\mathcal{D}}^{-}\left(x_{p}\right)$, coincide with all of its neighbors, denoted by $N_{\mathcal{D}}\left(x_{p}\right)$, where

$$
N_{\mathcal{D}}^{-}\left(x_{p}\right)=N_{\mathcal{D}}\left(x_{p}\right)=\left\{x:\left(x, x_{p}\right) \in E(\mathcal{D})\right\} \neq \varnothing .
$$

We can decompose the edge ideal of $\mathcal{D}$ as

$$
I(\mathcal{D})=I\left(\mathcal{D} \backslash x_{p}\right)+\underbrace{\left(x x_{p}^{w}: x \in N_{\mathcal{D}}\left(x_{p}\right)\right)}_{J}
$$

where $\mathcal{G}\left(I\left(\mathcal{D} \backslash x_{p}\right)\right)=\mathcal{G}(I(\mathcal{D})) \backslash \mathcal{G}(J)$.

Let $\mathcal{D}^{\prime}$ be a weight reduction of $\mathcal{D}$ such that

$$
I\left(\mathcal{D}^{\prime}\right)=I\left(\mathcal{D} \backslash x_{p}\right)+\underbrace{\left(x x_{p}^{w-1}: x \in N_{\mathcal{D}}\left(x_{p}\right)\right)}_{J^{\prime}} .
$$

Note that $\mathcal{D} \backslash x_{p}$ is an induced weighted oriented subgraph of $\mathcal{D}$ and $\mathcal{D}^{\prime}$.

Remark 15. In the light of Remark 7 , one can observe that for all $i>0$ and $\mathbf{b}=\left(b_{1}, \ldots, b_{n}\right) \in$ $\mathbb{N}^{n}$,

$$
\begin{aligned}
\beta_{i, \mathbf{b}}(I(\mathcal{D})) & =0 \text { if } b_{p} \neq 0, w, \\
\beta_{i, \mathbf{b}}\left(I\left(\mathcal{D}^{\prime}\right)\right) & =0 \text { if } b_{p} \neq 0, w-1, \\
\beta_{i, \mathbf{b}}\left(I\left(\mathcal{D} \backslash x_{p}\right)\right) & =0 \text { if } b_{p} \neq 0
\end{aligned}
$$

because the upper-Koszul complexes of these ideals at the corresponding multidegrees are all cones with apex $x_{p}$. 
Since only non-zero Betti numbers for the edge ideals of interest can occur at multidegrees $\mathbf{b} \in \mathbb{N}^{n}$ where $b_{p} \in\{0, w\}$ for $I(\mathcal{D})$ and $b_{p}^{\prime} \in\{0, w-1\}$ for $I\left(\mathcal{D}^{\prime}\right)$, we consider these two cases separately by finding relations between the corresponding upper-Koszul simplicial complexes.

Lemma 16. Let $\boldsymbol{b}=\left(b_{1}, \ldots, b_{n}\right), \boldsymbol{b}^{\prime}=\left(b_{1}^{\prime}, \ldots, b_{n}^{\prime}\right) \in \mathbb{N}^{n}$ such that $b_{i}^{\prime}=b_{i}$ for all $i \neq p$ and $b_{p}^{\prime}=b_{p}-1$.

(a) If $b_{p}=0$, then $\beta_{i, \boldsymbol{b}}(I(\mathcal{D}))=\beta_{i, \boldsymbol{b}}\left(I\left(\mathcal{D}^{\prime}\right)\right)=\beta_{i, \boldsymbol{b}}\left(I\left(\mathcal{D} \backslash x_{p}\right)\right)$.

(b) If $b_{p}=w$, then $\beta_{i, b}(I(\mathcal{D}))=\beta_{i, b^{\prime}}\left(I\left(\mathcal{D}^{\prime}\right)\right)$.

Proof. Given $F \subseteq\left\{x_{1}, \ldots, x_{n}\right\}$, we set $m:=\frac{x^{\mathbf{b}}}{x^{F}}$ and $m^{\prime}:=\frac{x^{\mathbf{b}^{\prime}}}{x^{F}}$ where $m^{\prime} x_{p}=m$.

(a) It suffices to show that the upper-Koszul simplicial complexes of $I(\mathcal{D}), I\left(\mathcal{D}^{\prime}\right)$ and $I\left(\mathcal{D} \backslash x_{p}\right)$ coincide at multidegree $\mathbf{b}$ by Theorem 6 . It is immediate from the chain of inclusions $I\left(\mathcal{D} \backslash x_{p}\right) \subseteq$ $I(\mathcal{D}) \subseteq I\left(\mathcal{D}^{\prime}\right)$ that

$$
K_{\mathbf{b}}\left(I\left(\mathcal{D} \backslash x_{p}\right)\right) \subseteq K_{\mathbf{b}}(I(\mathcal{D})) \subseteq K_{\mathbf{b}}\left(I\left(\mathcal{D}^{\prime}\right)\right) .
$$

Let $F \in K_{\mathbf{b}}(I(\mathcal{D}))$. Then $m \in I(\mathcal{D})$. Since $b_{p}=0$, none of the generators of $J$ divide $m$ and we must have $m \in I\left(\mathcal{D} \backslash x_{p}\right)$. Thus $F \in K_{\mathbf{b}}\left(I\left(\mathcal{D} \backslash x_{p}\right)\right)$, proving the equality $K_{\mathbf{b}}(I(\mathcal{D}))=$ $K_{\mathbf{b}}\left(I\left(\mathcal{D} \backslash x_{p}\right)\right)$. The remaining equality follows from the same arguments.

(b) It suffices to show that the upper-Koszul simplicial complex of $I(\mathcal{D})$ at multidegree $\mathbf{b}$ coincides with that of $I\left(\mathcal{D}^{\prime}\right)$ at multidegree $\mathbf{b}^{\prime}$.

Let $F \in K_{\mathbf{b}^{\prime}}\left(I\left(\mathcal{D}^{\prime}\right)\right)$. By the definition of upper-Koszul simplicial complexes, we have $m^{\prime} \in I\left(\mathcal{D}^{\prime}\right)$. If $m \in J^{\prime}$, then there exists $x x_{p}^{w-1} \in J^{\prime}$ such that $m^{\prime}$ is divisible by $x x_{p}^{w-1}$. As a result, $m=m^{\prime} x_{p} \in J$ and $m \in J \subseteq I(\mathcal{D})$ which implies that $F \in K_{\mathbf{b}}(I(\mathcal{D}))$. Suppose $m^{\prime} \notin J^{\prime}$. Then $m^{\prime}$ must be contained in $I\left(\mathcal{D} \backslash x_{p}\right)$, thus $m \in I\left(\mathcal{D} \backslash x_{p}\right) \subseteq I(\mathcal{D})$ and $F$ is a face in $K_{\mathbf{b}}(I(\mathcal{D}))$. It remains to prove the reverse containment.

If $F \in K_{\mathbf{b}}\left(I(\mathcal{D})\right.$ ), we have $m \in I(\mathcal{D})$. If $m \in J$, then $m$ must be divisible by some $x x_{p}^{w} \in J$ which implies that $x_{p} \notin F$. Since $w>1$, we have $m^{\prime} \in J^{\prime} \subseteq I\left(\mathcal{D}^{\prime}\right)$. Thus $F$ is a face in $K_{\mathbf{b}^{\prime}}\left(I\left(\mathcal{D}^{\prime}\right)\right)$. Suppose $m \notin J$. Then $m$ must be contained in $I\left(\mathcal{D} \backslash x_{p}\right)$. Equivalently, there exists a minimal generator $e$ of $I\left(\mathcal{D} \backslash x_{p}\right)$ such that $m=e \bar{m}$ for a monomial $\bar{m} \in R$. Since $w>1$, the monomial $\bar{m}$ is divisible by $x_{p}$ while $e$ is not. Thus $m^{\prime}$ is still contained in $I\left(\mathcal{D} \backslash x_{p}\right) \subseteq I\left(\mathcal{D}^{\prime}\right)$ and $F \in K_{\mathbf{b}^{\prime}}\left(I\left(\mathcal{D}^{\prime}\right)\right)$. Therefore, upper-Koszul simplicial complexes of $I(\mathcal{D})$ and $I\left(\mathcal{D}^{\prime}\right)$ agree at multidegrees $\mathbf{b}$ and $\mathbf{b}^{\prime}$.

Corollary 17. Let $\mathcal{D}$ be a weighted oriented graph with a sink vertex $x_{p}$ of weight $w>1$ and let $\mathcal{D}^{\prime}$ be the weight reduction of $\mathcal{D}$ on $x_{p}$. Then

$$
\operatorname{pdim}(R / I(\mathcal{D}))=\operatorname{pdim}\left(R / I\left(\mathcal{D}^{\prime}\right)\right) .
$$

Proof. It follows from showing the equality of the $i^{\text {th }}$ total Betti numbers of $I(\mathcal{D})$ and $I\left(\mathcal{D}^{\prime}\right)$ for all $i \geqslant 0$. Indeed, by Remark 15 and Lemma 16, we have

$$
\begin{aligned}
\beta_{i}(I(\mathcal{D}))=\sum_{\mathbf{b} \in \mathbb{N}^{n}} \beta_{i, \mathbf{b}}(I(\mathcal{D})) & =\sum_{\mathbf{b} \in \mathbb{N}^{n}, b_{p}=0} \beta_{i, \mathbf{b}}(I(\mathcal{D}))+\sum_{\mathbf{b} \in \mathbb{N}^{n}, b_{p}=w} \beta_{i, \mathbf{b}}(I(\mathcal{D})) \\
& =\sum_{\mathbf{b} \in \mathbb{N}^{n}, b_{p}=0} \beta_{i, \mathbf{b}}\left(I\left(\mathcal{D}^{\prime}\right)\right)+\sum_{\mathbf{b} \in \mathbb{N}^{n}, b_{p}=w-1} \beta_{i, \mathbf{b}}\left(I\left(\mathcal{D}^{\prime}\right)\right) \\
& =\sum_{\mathbf{b} \in \mathbb{N}^{n}} \beta_{i, \mathbf{b}}\left(I\left(\mathcal{D}^{\prime}\right)\right)=\beta_{i}\left(I\left(\mathcal{D}^{\prime}\right)\right) .
\end{aligned}
$$


One can question the affects of weight reduction process on regularity. Even though this process preserves the projective dimension when the reduction is done on a sink vertex of nonsimple weight, it has different impacts on regularity.

Example 18. Let $\mathcal{D}$ be a weighted oriented path with the edge ideal

$$
I(\mathcal{D})=\left(x_{2} x_{1}^{4}, x_{2} x_{3}, x_{3} x_{4}^{2}\right) .
$$

Let $\mathcal{D}^{\prime}$ be a weight reduction of $\mathcal{D}$ on $x_{4}$ such that $I\left(\mathcal{D}^{\prime}\right)=\left(x_{2} x_{1}^{4}, x_{2} x_{3}, x_{3} x_{4}\right)$ and $\mathcal{D}^{\prime \prime}$ be a weight reduction of $\mathcal{D}$ on $x_{1}$ such that $I\left(\mathcal{D}^{\prime \prime}\right)=\left(x_{2} x_{1}^{3}, x_{2} x_{3}, x_{3} x_{4}^{2}\right)$. Based on the Betti diagrams of $R / I(\mathcal{D}), R / I\left(\mathcal{D}^{\prime}\right)$ and $R / I\left(\mathcal{D}^{\prime \prime}\right)$ given below (in order) we have

$$
\operatorname{reg}(R / I(\mathcal{D}))=\operatorname{reg}\left(R / I\left(\mathcal{D}^{\prime}\right)\right)=4 \neq 3=\operatorname{reg}\left(R / I\left(\mathcal{D}^{\prime \prime}\right)\right) .
$$

Thus weight reduction process on $x_{4}$ preserves the regularity whereas weight reduction on $x_{1}$

\begin{tabular}{|c|c|c|}
\hline 0: & 1 & - \\
\hline 1: & - & 1 \\
\hline 2: & - & 1 \\
\hline 3: & - & - \\
\hline 4: & - & 1 \\
\hline
\end{tabular}
reduces the regularity by one. Note that total Betti numbers in each diagram are equal.

$\begin{array}{lccc} & 0 & 1 & 2 \\ ----------- \\ 0: & 1 & - & - \\ 1: & - & 2 & 1 \\ 2: & - & - & - \\ 3: & - & - & - \\ 4: & - & 1 & 1 \\ ------------ \\ \text { Tot : } & 1 & 3 & 2\end{array}$

$\begin{array}{lccc} & 0 & 1 & 2 \\ ------- & \\ 0: & 1 & - & - \\ 1: & - & 1 & - \\ 2: & - & 1 & 1 \\ 3: & - & 1 & 1 \\ & & \\ --------- \\ \text { Tot: } & 1 & 3 & 2\end{array}$

\section{Algebraic Invariants via Upper-Koszul Simplicial Complexes}

The main focus of this section is to obtain formulas for the projective dimension and regularity of edge ideals of weighted oriented graphs by exploiting the structure of related upper-Koszul simplicial complexes. Structure of an upper-Koszul simplicial complex heavily rely on the choice of a multidegree. An "optimal" choice for a multidegree can be achieved by encoding the weights of all vertices in the multidegree. We use the word optimal to emphasize that this particular multidegree can lead us to a unique extremal Betti number which in turn enables us to compute the projective dimension and the regularity.

By Hilbert's Syzygy Theorem, $\operatorname{pdim}(R / I) \leqslant n$ for any homogeneous ideal $I \subseteq R=$ $k\left[x_{1}, \ldots, x_{n}\right]$. It is well-known that this bound is tight. A famous example for this instance is the graded maximal ideal $\mathfrak{m}=\left(x_{1}, \ldots, x_{n}\right)$ as the Koszul complex on the variables $x_{1}, \ldots, x_{n}$ gives a minimal free resolution of $R / \mathfrak{m}$ of length $n$. In a recent paper [1], the class of monomial ideals with the largest projective dimension are characterized using dominant sets and divisibility conditions.

In the first result of this section, we characterize the structure of all weighted oriented graphs on $n$ vertices such that projective dimension of their edge ideals attain the largest possible value.

Theorem 19. Let $\mathcal{D}$ be a weighted oriented graph on the vertices $V(\mathcal{D})=\left\{x_{1}, \ldots, x_{n}\right\}$. Then

$$
\operatorname{pdim}(R / I(\mathcal{D}))=n
$$


if and only if there is an edge $e=\left(x_{j}, x_{i}\right)$ oriented towards $x_{i}$ for each $x_{i} \in V(\mathcal{D})$ such that $x_{j}$ has a non-simple weight. Furthermore,

$$
\operatorname{pdim}(R / I(\mathcal{D}))=n \text { if and only if } \beta_{n, \boldsymbol{b}}(R / I(\mathcal{D})) \neq 0
$$

where $\boldsymbol{b}=\left(w_{1}, \ldots, w_{n}\right) \in \mathbb{N}^{n}$.

Proof. Observe that $\operatorname{pdim}(R / I(\mathcal{D}))=n$ if and only if there exists a multidegree $\mathbf{a} \in \mathbb{N}^{n}$ such that $\beta_{n, \mathbf{a}}(R / I(\mathcal{D}))$ is non-zero. It follows from Theorem 6 that

$$
\beta_{n, \mathbf{a}}(R / I(\mathcal{D}))=\operatorname{dim}_{k} \widetilde{H}_{n-2}\left(K_{\mathbf{a}}(I(\mathcal{D}) ; k)\right) \neq 0
$$

which happens only when $F=\left\{x_{1}, \ldots, x_{n}\right\}$ is a minimal non-face of $K_{\mathbf{a}}(I(\mathcal{D}))$. Note that each $a_{i} \in\left\{0,1, w_{i}\right\}$. Otherwise, $\beta_{n, \mathbf{a}}(R / I(\mathcal{D}))=0$ by Remark 7 .

Let $\Delta=K_{\mathbf{a}}(I(\mathcal{D}))$ and $F_{i}:=F \backslash\left\{x_{i}\right\}$ for each $x_{i} \in F$. Recall that $F$ is a minimal non-face of $\Delta$ whenever $F \notin \Delta$ and each $F_{i} \in \Delta$. It follows from the definition of upper-Koszul simplicial complexes that

$$
\begin{aligned}
& F \notin \\
& F_{i} \Leftrightarrow \Delta \Longleftrightarrow m:=\frac{x^{\mathbf{a}}}{x^{F}}=\prod_{j=1}^{n} x_{j}^{a_{j}-1} \notin I(\mathcal{D}), \text { and } \\
& m_{i}:=\frac{x^{\mathbf{a}}}{x^{F_{i}}}=x_{i}^{a_{i}}\left(\prod_{j \neq i} x_{j}^{a_{j}-1}\right) \in I(\mathcal{D}) \text { for each } i \in\{1, \ldots, n\} .
\end{aligned}
$$

Note that each $a_{i} \neq 0$ when $m \notin I(\mathcal{D})$ and each $m_{i} \in I(\mathcal{D})$. The monomial $m_{i} \in I(\mathcal{D})$ if and only if there exists a minimal generator of $I(\mathcal{D})$ associated to an edge oriented towards $x_{i}$, say $e=\left(x_{k}, x_{i}\right)$, such that $x_{k} x_{i}^{w_{i}} \in I(\mathcal{D})$ divides $m_{i}$ for some $x_{k} \in V(\mathcal{D})$. This implies that $a_{i}=w_{i}$ and $a_{k}=w_{k}>1$. Therefore, $\operatorname{pdim}(R / I(\mathcal{D}))=n$ if and only if, for each $x_{i} \in V(\mathcal{D})$, there exists an edge $e=\left(x_{k}, x_{i}\right)$ oriented towards $x_{i}$ such that $w_{k}>1$. Notice that there are no source vertices when $\operatorname{pdim}(R / I(\mathcal{D}))=n$. The latter statement follows from the conclusion that $a_{i}=w_{i}$ for each $i=1, \ldots, n$ whenever $\beta_{n, \mathbf{a}}(R / I(\mathcal{D})) \neq 0$.

Remark 20. Let $\mathcal{D}$ be a weighted oriented graph on $n$ vertices. Let $\mathbf{b}=\left(w_{1}, \ldots, w_{n}\right) \in \mathbb{N}^{n}$ be a multidegree corresponding to the least common multiple of all minimal generators of $I(\mathcal{D})$ and $|\mathbf{b}|:=\sum_{i=1}^{n} w_{i}$. If $\beta_{p, \mathbf{b}}(R / I(\mathcal{D})) \neq 0$ for $p=\operatorname{pdim}\left(R / I(\mathcal{D})\right.$, then $\beta_{p,|\mathbf{b}|}(R / I(\mathcal{D}))$ is the unique extremal Betti number of $R / I(\mathcal{D})$. Because $\beta_{i, \mathbf{a}}(R / I(\mathcal{D}))=0$ for $\mathbf{a}=\left(a_{1}, \ldots, a_{n}\right) \in \mathbb{N}^{n}$ such that $a_{i}>w_{i}$ for some $i=1, \ldots, n$ by Remark 7 .

If $\mathcal{D}$ satisfies the condition given in the statement of Theorem 19, then $\beta_{n,|\mathbf{b}|}(R / I(\mathcal{D}))$ is the unique extremal Betti number of $R / I(\mathcal{D})$. In that case, we can further deduce the formula for the regularity of $R / I(\mathcal{D})$.

Corollary 21. Let $\mathcal{D}$ be a weighted oriented graph on $n$ vertices such that

$$
\operatorname{pdim}(R / I(\mathcal{D}))=n .
$$

Then

$$
\operatorname{reg}(R / I(\mathcal{D}))=\sum_{i=1}^{n} w_{i}-n
$$




\subsection{Weighted Oriented Cycles}

Let $\mathcal{D}$ be a weighted oriented cycle on $n$ vertices $x_{1}, \ldots, x_{n}$. We shall assume that there exists at least one vertex $x_{i}$ such that $w_{i}>1$ and there is an edge oriented into $x_{i}$. Otherwise, $\mathcal{D}$ can be considered as an unweighted, unoriented cycle whose Betti numbers are computed in [15]. If $\mathcal{D}$ has no sink vertices (or source vertices), then it must be endowed with the natural orientation (clockwise or counter clockwise). If all vertices of a naturally oriented weighted cycle $\mathcal{D}$ are of non-simple weight, its projective dimension and regularity can be computed by Theorem 19 and we recover one of the main results of [19].

Corollary 22. [19, Theorem 1.4.] Let $\mathcal{D}$ be naturally oriented weighted cycle on the vertices $\left\{x_{1}, \ldots, x_{n}\right\}$ where $w_{i}>1$ for all $i$. Then

$$
\operatorname{pdim}(R / I(\mathcal{D}))=n \text { and } \operatorname{reg}(R / I(\mathcal{D}))=\sum_{i=1}^{n} w_{i}-n .
$$

\subsection{Weighted Rooted Graphs}

In this subsection, we consider the possibility of having source vertices and their effects on algebraic invariants. In the existence of several source vertices, one needs more information on the structure of a weighted oriented graph to be able to compute its algebraic invariants. As a natural starting point, we consider weighted rooted graphs.

Definition 23. A weighted graph $\mathcal{D}$ is called rooted if there is a vertex distinguished as the root and there is a naturally oriented path (i.e., all edges on the path are in the same direction) from the root vertex to any other vertex in $\mathcal{D}$. Orientation of $\mathcal{D}$ is determined by naturally oriented paths from the root to other vertices. Note that the only source vertex of a weighted rooted graph is its root.

Remark 24. If $\mathcal{D}$ is a weighted rooted graph, it does not fit in the description of weighted oriented graphs whose edge ideals have the largest projective dimension in Theorem 19. Thus $\operatorname{pdim}(R / I(\mathcal{D})) \leqslant n-1$.

Theorem 25. Let $\mathcal{D}$ be a weighted rooted graph on the vertices $\left\{x_{1}, \ldots, x_{n}\right\}$ with the root vertex $x_{1}$. Suppose $w_{i} \geqslant 2$ for all $i \neq 1$. Then

$$
\operatorname{pdim}(R / I(\mathcal{D}))=n-1 \text { and } \operatorname{reg}(R / I(\mathcal{D}))=\sum_{i=1}^{n} w_{i}-n+1
$$

Proof. Let $\mathbf{b}=\left(1, w_{2}, \ldots, w_{n}\right) \in \mathbb{N}^{n}$. It suffices to show that $\beta_{n-1,|\mathbf{b}|}(R / I(\mathcal{D}))$ is the unique extremal Betti number of $R / I(\mathcal{D})$.

Consider the upper-Koszul complex $K_{\mathbf{b}}(I(\mathcal{D}))$ of $I(\mathcal{D})$. Let $F=\left\{x_{2}, \ldots, x_{n}\right\}$ and $F_{i}=$ $F \backslash\left\{x_{i}\right\}$ for $2 \leqslant i \leqslant n$. Our goal is to show that $F$ is a minimal non-face of $K_{\mathbf{b}}(I(\mathcal{D}))$. First observe that $F \notin K_{\mathbf{b}}(I(\mathcal{D})$ ). Otherwise, by the definition of upper-Koszul simplicial complexes, we must have $x_{1} \prod_{i=2}^{n} x_{i}^{w_{i}-1} \in I(\mathcal{D})$ which is not possible because each generator of $I(\mathcal{D})$ must be divisible by $x_{i}^{w_{i}}$ for some $i \in\{2, \ldots, n\}$.

If each $F_{i} \in K_{\mathbf{b}}(I(\mathcal{D}))$, then $F$ must be a minimal non-face. Note that

$$
m_{i}:=\frac{\prod_{j=1}^{n} x_{j}^{w_{j}}}{x_{2} \cdots \hat{x}_{i} \cdots x_{n}}=x_{1}\left(\prod_{i=1, j \neq i}^{n} x_{j}^{w_{j}-1}\right) x_{i}^{w_{i}} \in I(\mathcal{D})
$$


because all non-source vertices have non-simple weights and $m_{i}$ is divisible by $x_{1} x_{i}^{w_{i}} \in I(\mathcal{D})$ or $x_{j} x_{i}^{w_{i}} \in I(\mathcal{D})$ for some $i \in\{2, \ldots, n\}$. It implies that $F_{i} \in K_{\mathbf{b}}(I(\mathcal{D}))$ for each $i$. Thus $F$ is an $(n-2)$-dimensional minimal non-face of $K_{\mathbf{b}}(I(\mathcal{D}))$. It follows from Theorem 6 that

$$
\left.\beta_{n-1, \mathbf{b}}(R / I(\mathcal{D}))=\operatorname{dim}_{k} \widetilde{H}_{n-3}\left(K_{\mathbf{b}}(I(\mathcal{D})) ; k\right)\right) \neq 0 .
$$

Therefore, $\beta_{n-1,|\mathbf{b}|}(R / I(\mathcal{D}))$ is the unique extremal Betti number of $R / I(\mathcal{D})$ by Theorem 19 and Remark 20.

As an immediate consequence of Theorem 25, we recover several results from [19].

Corollary 26. [19, Theorem 1.2. and Theorem 1.3.] Let $\mathcal{D}$ be weighted rooted forest with non-simple weights. Then

$$
\operatorname{pdim}(R / I(\mathcal{D}))=n-1 \text { and } \operatorname{reg}(R / I(\mathcal{D}))=\sum_{i=1}^{n} w_{i}-n+1 .
$$

\section{$5 \quad$ Weighted Oriented Complete Graphs}

The objects of this section are weighted oriented complete graphs. Throughout this section, we may assume that each weighted oriented complete graph has at least one vertex of non-simple weight. Otherwise, it is the unweighted, unoriented complete graph and Betti numbers of its edge ideal are well-understood from its independence complex due to Hochster's formula.

Definition 27. Let $\mathcal{D}$ be a weighted oriented complete graph on the vertices $\left\{x_{1}, \ldots, x_{n}\right\}$. We call $\mathcal{D}$ a naturally oriented complete graph if the oriented edge set is given by $\left\{\left(x_{i}, x_{j}\right): 1 \leqslant\right.$ $i<j \leqslant n\}$. Then the edge ideal of $\mathcal{D}$ is

$$
I(\mathcal{D})=\left(x_{i} x_{j}^{w_{j}}: 1 \leqslant i<j \leqslant n\right) .
$$

Since $x_{1}$ is a source vertex, we set $w_{1}=1$.

In what follows, we provide formulas for the projective dimension and the regularity for the edge ideal of a naturally oriented weighted complete graph $\mathcal{D}$. The key ingredient of the proof is the use of upper-Koszul simplicial complex of $I(\mathcal{D})$.

Theorem 28. Let $\mathcal{D}$ be a naturally oriented weighted complete graph on the vertices $\left\{x_{1}, \ldots, x_{n}\right\}$ such that $w_{p}>1$ for some $p \geqslant 2$. Then

$$
\operatorname{pdim}(R / I(\mathcal{D}))=n-1 \text { and } \operatorname{reg}(R / I(\mathcal{D}))=\sum_{i=1}^{n} w_{i}-n+1
$$

Proof. Let $\mathbf{b}=\left(w_{1}, w_{2}, \ldots, w_{n}\right) \in \mathbb{N}^{n}$. We claim that $\beta_{n-1, \mathbf{b}}(R / I(\mathcal{D}))$ is the unique extremal Betti number of $R / I(\mathcal{D})$. As an immediate consequence of the claim, we obtain the expressions given in the statement of the theorem as the values of the projective dimension and the regularity of $R / I(\mathcal{D})$.

In order to prove the claim, consider the upper Koszul simplicial complex of $I(\mathcal{D})$ in multidegree $\mathbf{b}$ and denote it by $\Delta:=K_{\mathbf{b}}(I(\mathcal{D}))$. For the first part of the claim, it suffices to show that the only minimal non-faces of $\Delta$ are $(n-2)$-dimensional. 
Let $F=\left\{x_{1}, \ldots, x_{n}\right\}$ and $F_{1}=F \backslash\left\{x_{1}\right\}$. Since $x^{\mathbf{b}-F}=\prod_{i=2}^{n} x_{i}^{w_{i}-1}$ and $x^{\mathbf{b}-F_{1}}=$ $x_{1} \prod_{i=2}^{n} x_{i}^{w_{i}-1}$ are not contained in $I(\mathcal{D})$, neither $F$ nor $F_{1}$ is a face in $\Delta$. In addition, let $F_{i, j}:=F \backslash\left\{x_{i}, x_{j}\right\}$ for each $1 \leqslant i<j \leqslant n$. Observe that each $F_{i, j}$ is a face in $\Delta$ because

$$
\frac{x^{\mathbf{b}}}{x^{F_{i, j}}}=\left(\prod_{k \neq i, j} x_{k}^{w_{k}-1}\right) x_{i}^{w_{i}} x_{j}^{w_{j}} \in I(\mathcal{D}) .
$$

Therefore, the upper Koszul simplicial complex $\Delta$ has at least one minimal non-face of dimension $(n-2)$. Then, we have $\left.\operatorname{dim}_{k} \widetilde{H}_{n-3}(\Delta ; k)\right) \neq 0$. Hence, by Theorem 6 ,

$$
\left.\beta_{n-1, \mathbf{b}}(R / I(\mathcal{D}))=\beta_{n-2, \mathbf{b}}(I(\mathcal{D}))=\operatorname{dim}_{k} \widetilde{H}_{n-3}(\Delta ; k)\right) \neq 0 .
$$

Note that $\mathcal{D}$ does not belong to the class of graphs expressed in Theorem 19 and it follows that $\operatorname{pdim}(R / I(\mathcal{D})) \leqslant n-1$. Thus, $\beta_{n-1,|\mathbf{b}|}(R / I(\mathcal{D}))$ is the unique extremal Betti number of $R / I(\mathcal{D})$ by Remark 20 .

Remark 29. Recall that, when $w_{i} \geqslant 2$ for all $x_{i} \neq x_{1}$, the edge ideal $I(\mathcal{D})$ is the initial ideal of the vanishing ideal of a projective nested cartesian set. As mentioned in the introduction, $\operatorname{reg}(R / I(\mathcal{D}))$ is a strict upper bound for the degree of the evaluation map used in creating projective nested cartesian codes. It was shown in [8] that degree of the evaluation map must be less than $\sum_{i=1}^{n} w_{i}-n+1$ for a projective nested cartesian code to have an "optimal" minimum distance ([8, Theorem 3.8]). However, this upper bound is not obtained by computing $\operatorname{reg}(R / I(\mathcal{D}))$ explicitly in $[8]$ and the equality $\operatorname{reg}(R / I(\mathcal{D}))=\sum_{i=1}^{n} w_{i}-n+1$ is rather concluded from their results (see [16, Proposition 6.3]). In a way, Theorem 28 part (b) recovers this embedded conclusion.

Above theorem completes the discussion of regularity and projective dimension of $I(\mathcal{D})$ when $\mathcal{D}$ is naturally oriented. If the orientation of a weighted complete graph is not known, finding regularity and projective dimension through upper-Koszul simplicial complexes becomes a more difficult task. In the absence of an explicit orientation, structure of the upper-Koszul simplicial complex of $I(\mathcal{D})$ is more complex. Thus, one needs to employ different techniques than upper-Koszul simplicial complexes.

In the following result, we provide a recursive formula for the Betti numbers of $I(\mathcal{D})$ in the existence of a sink vertex. This condition can be considered as a local property of $\mathcal{D}$. Note that if there exists at least one sink vertex, by relabeling the vertices, we may assume that $x_{n}$ is a sink.

Theorem 30. Let $\mathcal{D}_{n}$ be a weighted oriented complete graph on the vertices $\left\{x_{1}, \ldots, x_{n}\right\}$. If $x_{n}$ is a sink in $\mathcal{D}_{n}$, we have

$$
\beta_{i, j}\left(R / I\left(\mathcal{D}_{n}\right)\right)= \begin{cases}\beta_{i, j}\left(R / I\left(\mathcal{D}_{n-1}\right)\right)+\left(\begin{array}{c}
n-1 \\
i
\end{array}\right)+\beta_{i-1, j-w_{n}}\left(R / I\left(\mathcal{D}_{n-1}\right)\right) & : j=i+w_{n} \\
\beta_{i, j}\left(R / I\left(\mathcal{D}_{n-1}\right)\right)+\beta_{i-1, j-w_{n}}\left(R / I\left(\mathcal{D}_{n-1}\right)\right) & : j \neq i+w_{n}\end{cases}
$$

for all $i>1$ where $\mathcal{D}_{n-1}$ is obtained from $\mathcal{D}_{n}$ by deleting the vertex $x_{n}$.

Proof. Let $J=\left(x_{i} x_{n}^{w_{n}}: 1 \leqslant i<n\right)$. Then one can decompose $I(\mathcal{D})$ as a disjoint sum of $I\left(\mathcal{D}_{n}\right)=$ $I\left(\mathcal{D}_{n-1}\right)+J$ where $\mathcal{D}_{n-1}=\mathcal{D}_{n} \backslash x_{n}$ is a weighted oriented complete graph on $(n-1)$ vertices. It is clear that $\mathcal{D}_{n-1}$ is an induced weighted oriented subgraph of $\mathcal{D}_{n}$. Note that the minimal free resolution of $J$ is obtained from shifting the minimal free resolution of $R /\left(x_{1}, \ldots, x_{n-1}\right)$ 
by degree $w_{n}$. Thus $J$ has a linear resolution. It then follows from Theorem 9 that $I\left(\mathcal{D}_{n}\right)=$ $I\left(\mathcal{D}_{n-1}\right)+J$ is a Betti splitting because $\mathcal{G}(J)$ contains all the generators of $I(\mathcal{D})$ divisible by $x_{n}$. Then, by Definition 8 ,

$$
\beta_{i, j}\left(R / I\left(\mathcal{D}_{n}\right)\right)=\beta_{i, j}\left(R / I\left(\mathcal{D}_{n-1}\right)\right)+\beta_{i, j}(R / J)+\beta_{i-1, j}\left(R / I\left(\mathcal{D}_{n-1}\right) \cap J\right)
$$

for $i>1$. Our goal is to analyze each term of the above expression.

It is immediate from the definition of $J$ that $\beta_{i, j+w_{n}}(R / J)=\beta_{i, j}\left(R /\left(x_{1}, \ldots, x_{n-1}\right)\right)$ for $i>0$. Recall that Koszul complex is a minimal free resolution of the $R$-module $R /\left(x_{1}, \ldots, x_{n-1}\right)$ and the only non-zero Betti numbers occur when $j=i$. In particular, for $1 \leqslant i \leqslant n-1$, we have

$$
\beta_{i, i}\left(R /\left(x_{1}, \ldots, x_{n-1}\right)\right)=\left(\begin{array}{c}
n-1 \\
i
\end{array}\right)=\beta_{i, i+w}(R / J)
$$

as the only non-zero Betti numbers of $J$.

Next, observe that $I\left(\mathcal{D}_{n-1}\right) \cap J=\left(x_{n}^{w_{n}}\right) I\left(\mathcal{D}_{n-1}\right)$. Then, we can express the Betti numbers of the intersection in terms of iterated Betti numbers of $I\left(\mathcal{D}_{n-1}\right)$. More specifically, for all $i>0$,

$$
\beta_{i, j+w}\left(R / I\left(\mathcal{D}_{n-1}\right) \cap J\right)=\beta_{i, j}\left(R / I\left(\mathcal{D}_{n-1}\right)\right) .
$$

Therefore, one can obtain the expressions given in the statement of the theorem by using Equation (5.1) and Equation (5.2).

Corollary 31. Let $\mathcal{D}_{n}$ be a weighted oriented complete graph on the vertices $\left\{x_{1}, \ldots, x_{n}\right\}$. If $x_{n}$ is a sink in $\mathcal{D}_{n}$, then

$$
\operatorname{pdim}\left(R / I\left(\mathcal{D}_{n}\right)\right) \in\{n-1, n\} \text { and } \operatorname{reg}\left(R / I\left(\mathcal{D}_{n}\right)\right)=\operatorname{reg}\left(R / I\left(\mathcal{D}_{n-1}\right)\right)+\left(w_{n}-1\right) .
$$

Proof. Let $p=\operatorname{pdim}\left(R / I\left(\mathcal{D}_{n-1}\right)\right)$ and $\left.r=\operatorname{reg}\left(R / I\left(\mathcal{D}_{n-1}\right)\right)\right)$. Then $\beta_{p, p+r}\left(R / I\left(\mathcal{D}_{n-1}\right)\right) \neq 0$ and $\beta_{i, j}\left(R / I\left(\mathcal{D}_{n-1}\right)\right)=0$ for $i>p$ or $j>p+r$. By using Theorem 30, we obtain the following top non-zero Betti numbers of $R / I\left(\mathcal{D}_{n}\right)$.

$$
\begin{aligned}
& \beta_{p+1, p+r+w_{n}}\left(R / I\left(\mathcal{D}_{n}\right)\right) \neq 0 \\
& \beta_{n-1, n-1+w_{n}}\left(R / I\left(\mathcal{D}_{n}\right)\right) \neq 0
\end{aligned}
$$

Hence, Equation (5.3) and Equation (5.4) imply that

$$
\begin{aligned}
\operatorname{reg}\left(R / I\left(\mathcal{D}_{n}\right)\right) & =\max \left\{w_{n}, r+w_{n}-1\right\}=r+w_{n}-1 \\
& =\operatorname{reg}\left(R / I\left(\mathcal{D}_{n-1}\right)\right)+\left(w_{n}-1\right) .
\end{aligned}
$$

Similarly, using the top non-zero Betti numbers, we have

$$
\operatorname{pdim}\left(R / I\left(\mathcal{D}_{n}\right)\right)=\max \{n-1, p+1\}
$$

Since $p \leqslant n-1$, the projective dimension is either $n-1$ or $n$.

Remark 32. If the underlying graph of a weighted oriented graph $\mathcal{D}$ on the vertices $\left\{x_{1}, \ldots, x_{n}\right\}$ is a star, we call $\mathcal{D}$ a weighted oriented star graph. Let $x_{n}$ be the center of $\mathcal{D}$. If $x_{n}$ is a sink vertex, we say $\mathcal{D}$ is a weighted oriented star with a center sink. The edge ideal of a weighted oriented star with a center sink $x_{n}$ is given as

$$
I(\mathcal{D})=\left(x_{i} x_{n}^{w_{n}}: 1 \leqslant i \leqslant n-1\right) .
$$

As discussed in the proof of Theorem 30, the module $R / I(\mathcal{D})$ has a linear resolution and it is obtained by shifting the Koszul complex of $R /\left(x_{1}, \ldots, x_{n-1}\right)$ by degree $w_{n}$. Then

$$
\operatorname{pdim}(R / I(\mathcal{D}))=n-1 \text { and } \operatorname{reg}(R / I(\mathcal{D}))=w_{n} .
$$




\section{Betti Numbers via Mapping Cone Construction}

In this section, we provide a recursive formula for the Betti numbers of edge ideals of weighted oriented graphs with at least one leaf vertex which is also a sink. We achieve it by employing a technique called the mapping cone construction. This technique is different than Betti splittings while being as powerful.

Recall that Betti splitting is a method which allows one to express Betti numbers of an ideal in terms of smaller ideals. In a similar vein, mapping cone construction allows one to build a free resolution of an $R$-module $M$ in terms of $R$-modules associated to $M$. In particular, given a short exact sequence

$$
0 \longrightarrow R / M^{\prime} \longrightarrow R / M^{\prime \prime} \longrightarrow R / M \longrightarrow 0
$$

where $M^{\prime}, M^{\prime \prime}$ and $M$ are graded $R$-modules, the mapping cone construction provides a free resolution of $M$ in terms of free resolutions of $M^{\prime}$ and $M^{\prime \prime}$. For more details on the mapping cone construction, we refer the reader to [17]. In general, given minimal free resolutions for $M^{\prime}$ and $M^{\prime \prime}$, the mapping cone construction does not necessarily give a minimal free resolution of $M$. However, there are classes of ideals in which the mapping cone construction provides a minimal free resolution for particular short exact sequences. In particular, the case of edge ideal of an unweighted, unoriented graph is studied in [6, 2.1.1. Theorem] and the case of path ideals of rooted trees is investigated in $[7,2.7$. Theorem]. In what follows, we extend these results to the case of weighted oriented graphs using similar ideas.

Let $\mathcal{D}$ be a weighted oriented graph with the vertex set $V(\mathcal{D})=\left\{x_{1}, \ldots, x_{n}\right\}$. A vertex is called a leaf if there is only one edge incident to it. In the existence of at least one leaf vertex which is also a sink, one can use the mapping cone construction to describe Betti numbers of $R / I(\mathcal{D})$ recursively. Note that there is no restriction on the overall orientation of $\mathcal{D}$.

Theorem 33. Let $\mathcal{D}$ be a weighted oriented graph on the vertices $\left\{x_{1}, \ldots, x_{n}\right\}$ with a leaf $x_{n}$. Suppose $x_{n}$ is a sink vertex. Then the mapping cone construction applied to the short exact sequence

$$
0 \longrightarrow \frac{R}{I\left(\mathcal{D} \backslash x_{n}\right):\left(x_{n-1} x_{n}^{w_{n}}\right)}\left(-w_{n}-1\right) \stackrel{x_{n-1} x_{n}^{w_{n}}}{\longrightarrow} \frac{R}{I\left(\mathcal{D} \backslash x_{n}\right)} \longrightarrow \frac{R}{I(\mathcal{D})} \longrightarrow 0
$$

provides a minimal free resolution of $R / I(\mathcal{D})$. In particular, for any $i$ and $j$, we have

$$
\left.\beta_{i, j}(R / I(\mathcal{D}))=\beta_{i, j}\left(R / I\left(\mathcal{D} \backslash x_{n}\right)\right)+\beta_{i-1, j-w-1}\left(R / I\left(\mathcal{D} \backslash x_{n}\right): x_{n-1}\right)\right) .
$$

Proof. Let $\mathcal{D}^{\prime}$ denote the weighted oriented induced subgraph $\mathcal{D} \backslash x_{n}$ of $\mathcal{D}$ and let $x_{n-1}$ be the unique neighbor of $x_{n}$ such that $\left(x_{n-1}, x_{n}\right) \in E(\mathcal{D})$. Since $x_{n}^{w_{n}}$ does not divide a minimal generator of $I\left(\mathcal{D}^{\prime}\right)$, one has

$$
I\left(\mathcal{D}^{\prime}\right):\left(x_{n-1} x_{n}^{w_{n}}\right)=I\left(\mathcal{D}^{\prime}\right): x_{n-1} .
$$

Then, it implies that the exact sequence

$$
0 \longrightarrow \frac{R}{\left.I\left(\mathcal{D}^{\prime}\right):\left(x_{n-1} x_{n}^{w_{n}}\right)\right)}\left(-w_{n}-1\right) \stackrel{x_{n-1} x_{n}^{w_{n}}}{\delta} \frac{R}{I\left(\mathcal{D}^{\prime}\right)} \longrightarrow \frac{R}{I(\mathcal{D})} \longrightarrow 0
$$

factors as

$$
\begin{gathered}
0 \longrightarrow \frac{R}{I\left(\mathcal{D}^{\prime}\right):\left(x_{n-1} x_{n}^{w_{n}}\right)}\left(-w_{n}-1\right) \stackrel{x_{n-1} x_{n}^{w_{n}}}{\longrightarrow} \frac{R}{I\left(\mathcal{D}^{\prime}\right)} \longrightarrow \frac{R}{I(\mathcal{D})} \longrightarrow 0 . \\
\qquad \begin{array}{c}
x_{n}^{w_{n}} \\
R / I\left(\mathcal{D}^{\prime}\right): x_{n-1}
\end{array}
\end{gathered}
$$


Let

$$
\begin{array}{ll}
\mathcal{F}: & 0 \cdots \stackrel{\phi_{2}}{\longrightarrow} F_{1} \stackrel{\phi_{1}}{\longrightarrow} F_{0}=R \stackrel{\phi_{0}}{\longrightarrow} R / I\left(\mathcal{D}^{\prime}\right):\left(x_{n-1} x_{n}^{w_{n}}\right) \longrightarrow 0 \text { and } \\
\mathcal{G}: & 0 \cdots \stackrel{\psi_{2}}{\longrightarrow} G_{1} \stackrel{\psi_{1}}{\longrightarrow} G_{0}=R \stackrel{\psi_{0}}{\longrightarrow} R / I\left(\mathcal{D}^{\prime}\right) \longrightarrow 0
\end{array}
$$

be minimal free resolutions of $R / I\left(\mathcal{D}^{\prime}\right):\left(x_{n-1} x_{n}^{w_{n}}\right)$ and $R / I\left(\mathcal{D}^{\prime}\right)$, respectively. Then the mapping construction applied to Equation (6.1) provides a free resolution of $R / I(\mathcal{D})$ given by

$$
0 \cdots \stackrel{\varphi_{3}}{\longrightarrow} G_{2} \oplus F_{1}\left(-w_{n}-1\right) \stackrel{\varphi_{2}}{\longrightarrow} G_{1} \oplus R\left(-w_{n}-1\right) \stackrel{\varphi_{1}}{\longrightarrow} R \stackrel{\varphi_{0}}{\longrightarrow} R / I(\mathcal{D}) \longrightarrow 0
$$

where the map $\varphi_{i}$ 's are defined by $\varphi_{1}=\left[\begin{array}{ll}\psi_{1} & -\delta_{0}\end{array}\right]$ and

$$
\varphi_{i}=\left[\begin{array}{cc}
\psi_{i} & (-1)^{i} \delta_{i-1} \\
0 & \phi_{i-1}
\end{array}\right]
$$

for $i>1$ such that each $\delta_{i}: F_{i}\left(-w_{n}-1\right) \longrightarrow G_{i}$ is induced from the homomorphism $\delta$.

It follows from the factorization in Equation (6.2) that the entries of the matrix of $\delta_{i}$ are not units. Since $\mathcal{F}$ and $\mathcal{G}$ are minimal free resolutions, then none of the entries in the matrix representation of $\varphi_{i}$ can be units. Thus the mapping cone construction applied to Equation (6.1) results with a minimal free resolution of $R / I(\mathcal{D})$. In particular, this implies the following recursive formula for the Betti numbers of $R / I(\mathcal{D})$

$$
\beta_{i, j}(R / I(\mathcal{D}))=\beta_{i, j}\left(R / I\left(\mathcal{D}^{\prime}\right)\right)+\beta_{i-1, j-w_{n}-1}\left(R / I\left(\mathcal{D}^{\prime}\right): x_{n-1}\right)
$$

for any $i, j$.

Corollary 34. Let $\mathcal{D}$ be a weighted oriented graph on the vertices $\left\{x_{1}, \ldots, x_{n}\right\}$ such that $x_{n}$ is a leaf and a sink vertex. Then

(a) $\operatorname{reg}(R / I(\mathcal{D}))=\max \left\{\operatorname{reg}\left(R / I\left(\mathcal{D} \backslash x_{n}\right)\right), \operatorname{reg}\left(R / I\left(\mathcal{D} \backslash x_{n}\right): x_{n-1}\right)+1\right\}$ and

(b) $\operatorname{pdim}(R / I(\mathcal{D}))=\max \left\{\operatorname{pdim}\left(R / I\left(\mathcal{D} \backslash x_{n}\right)\right), \operatorname{pdim}\left(R / I\left(\mathcal{D} \backslash x_{n}\right): x_{n-1}\right)+1\right\}$.

\subsection{Application}

Let $\mathcal{D}_{n}$ denote a weighted naturally oriented path on $n$ vertices. If all non-source vertices have non-simple weights, regularity and projective dimension formulas follow from Corollary 26. If one allows non-source vertices to have simple weights, computing the regularity and the projective dimension becomes a much more complicated task as these invariants heavily rely on the orientation of the graph and the positions of non-simple weights. Providing formulas for the regularity and projective dimension of any weighted oriented graph is an open problem.

In an attempt to address this general problem, weighted naturally oriented paths and cycles are studied in [5]. Indeed, positions of non-simple weights is quite crucial in computing the regularity (see [5, Theorem 5.9]). Particularly, whenever there are consecutive non-simple weight vertices $x_{i}$ and $x_{i+2}$ such that $x_{i+1}$ has a simple weight, then $x_{i}$ and $x_{i+2}$ can not "contribute" to the regularity simultaneously. One needs to consider the contribution of the one or the other and determine the regularity by taking the maximums of corresponding contributions (see [5, Notation 5.4, Definition 5.6 and Theorem 5.9]).

In what follows, we consider a more general case than that of [5] and provide a recursive formula for the Betti numbers of egde ideal of a weighted oriented path. Furthermore, our recursive formulas can offer an explanation about the "distance two away condition" of $[5$, Theorem 5.9]. 
Corollary 35. Let $\mathcal{D}_{n}$ denote a weighted oriented path on the vertices $x_{1}, \ldots, x_{n}$ and $\mathcal{D}_{n}^{\prime}$ be a weight reduction of $\mathcal{D}_{n}$. Suppose $\left(x_{n-2}, x_{n-1}\right),\left(x_{n-1}, x_{n}\right) \in E\left(\mathcal{D}_{n}\right)$.

(a) If $x_{n-1}$ is a non-trivial vertex, then

$$
\operatorname{reg}\left(R / I\left(\mathcal{D}_{n}\right)\right)=\max \left\{\operatorname{reg}\left(R / I\left(\mathcal{D}_{n-1}\right)\right), \operatorname{reg}\left(R / I\left(\mathcal{D}_{n-1}^{\prime}\right)\right)+w_{n}\right\} .
$$

(b) If $x_{n-1}$ is a trivial vertex, then

$$
\operatorname{reg}\left(R / I\left(\mathcal{D}_{n}\right)\right)=\max \left\{\operatorname{reg}\left(R / I\left(\mathcal{D}_{n-1}\right)\right), \operatorname{reg}\left(R / I\left(\mathcal{D}_{n-3}\right)\right)+w_{n}\right\} .
$$

Proof. (a) Suppose $w_{n-1}>1$. Then $I\left(\mathcal{D}_{n-1}\right): x_{n-1}=I\left(\mathcal{D}_{n-1}^{\prime}\right)$ where $\mathcal{D}_{n-1}^{\prime}$ is a weight reduction of $\mathcal{D}_{n-1}$ on $x_{n-1}$. Thus, it follows from Corollary 34 that

$$
\operatorname{reg}\left(R / I\left(\mathcal{D}_{n}\right)\right)=\max \left\{\operatorname{reg}\left(R / I\left(\mathcal{D}_{n-1}\right)\right), \operatorname{reg}\left(R / I\left(\mathcal{D}_{n-1}^{\prime}\right)\right)+w_{n}\right\} .
$$

(b) Suppose $w_{n-1}=1$. Let $I^{\prime}:=I\left(\mathcal{D}_{n-1}\right): x_{n-1}=I\left(\mathcal{D}_{n-3}\right)+\left(x_{n-2}\right)$. Since $x_{n-2}$ does not divide any minimal generator of $I\left(\mathcal{D}_{n-3}\right)$, one can obtain the minimal free resolution of $R / I^{\prime}$ by taking the tensor product of minimal free resolutions of $R / I\left(\mathcal{D}_{n-3}\right)$ and $R /\left(x_{n-2}\right)$. Then

$$
\operatorname{reg}\left(R / I^{\prime}\right)=\operatorname{reg}\left(R / I\left(\mathcal{D}_{n-3}\right)\right),
$$

and the statement follows from Corollary 34 .

Remark 36. Let $\mathcal{D}_{n}$ be a weighted naturally oriented path on the vertices $x_{1}, \ldots, x_{n}$. If $w_{n}>$ 1 and $w_{n-1}=1$, we can use Corollary 35 part (b) to determine the regularity of $R / I\left(\mathcal{D}_{n}\right)$ inductively by taking the maximum of the following two expressions.

$$
\operatorname{reg}\left(R / I\left(\mathcal{D}_{n}\right)\right)=\max \left\{\operatorname{reg}\left(R / I\left(\mathcal{D}_{n-1}\right)\right), \operatorname{reg}\left(R / I\left(\mathcal{D}_{n-3}\right)\right)+w_{n}\right\}
$$

Note that the ideal in the first expression contains $x_{n-2}$ in its support. However, vertex $x_{n}$ is not in the support of the first ideal and its weight does not contribute to the regularity in the first expression. On the other hand, the second expression contains $w_{n}$, the weight contribution of $x_{n}$, and the ideal associated to it does not contain $x_{n-2}$ in its support. Thus, Corollary 35 part (b) exhibits the behavior of distance two away vertices of non-simple weights in the regularity computations.

\section{Concluding Remarks}

In the final section of the paper, we present a useful observation relating some of the algebraic invariants of edge ideals of weighted oriented graphs and their underlying graphs.

Observation 37. Let $\mathcal{D}$ be a weighted oriented graph on the vertices $\left\{x_{1}, \ldots, x_{n}\right\}$ and $G$ be its underlying graph with their edge ideals $I(\mathcal{D})$ and $I(G)$ in $R=k\left[x_{1}, \ldots, x_{n}\right]$. If all vertices of $D$ are of simple weight, namely, $w_{i}=1$ for each vertex $x_{i}$, then $I(\mathcal{D})$ and $I(G)$ coincide. Suppose $\mathcal{D}$ has at least one vertex of non-simple weight. Let $S=R\left[y_{1,2}, \ldots, y_{1, w_{1}}, \ldots y_{n, 2}, \ldots, y_{n, w_{n}}\right]$ and $I \subset S$ be a polarization of $I(\mathcal{D})$. Recall that polarization preserves the graded Betti numbers.

Let $\mathbb{F}$ denote the minimal free resolution of $S / I$. We use $\mathbb{F}$. denote the localization of $\mathbb{F}$ at the variables $y_{i, j}$ 's where $j=2, \ldots, w_{i}$ for $i=1, \ldots, n$. Note that $\mathbb{F} \bullet$ is a free resolution (not necessarily minimal) of $R / I(G)$. In particular, $\mathbb{F} \bullet$ is obtained from $\mathbb{F}$ by setting $y_{i, j}=1$ for each $y_{i, j}$ in the differentials of $\mathbb{F}$. As a result, one has 
(a) $\beta_{i}(R / I(G)) \leqslant \beta_{i}(R / I(\mathcal{D}))$ for all $i \geqslant 0$,

(b) $\operatorname{pdim}(R / I(G)) \leqslant \operatorname{pdim}(R / I(\mathcal{D}))$, and

(c) $\operatorname{reg}(R / I(G)) \leqslant \operatorname{reg}(R / I(\mathcal{D}))$.

We close this section with the following question which is motivated by Corollary 17 .

Question 38. Let $\mathcal{D}$ be a weighted oriented graph with a non-simple weight vertex $x_{i}$ and let $\mathcal{D}^{\prime}$ be a weight reduction of $\mathcal{D}$ on $x_{i}$.

(a) When is $\beta_{i}(R / I(\mathcal{D}))=\beta_{i}\left(R / I\left(\mathcal{D}^{\prime}\right)\right)$ for all $i \geqslant 0$ ?

(b) Is there any relation between $\beta_{i, j}(R / I(\mathcal{D}))$ and $\beta_{i, j}\left(R / I\left(\mathcal{D}^{\prime}\right)\right)$ ?

(c) When is $\operatorname{pdim}(R / I(\mathcal{D}))=\operatorname{pdim}\left(R / I\left(\mathcal{D}^{\prime}\right)\right)$ ?

(d) When is $\operatorname{reg}(R / I(\mathcal{D}))=\operatorname{reg}\left(R / I\left(\mathcal{D}^{\prime}\right)\right)+1$ ?

Example 39. Let $I(\mathcal{D})=\left(x_{2} x_{1}, x_{3} x_{2}^{3}, x_{4} x_{3}^{2}, x_{4} x_{5}\right)$. Consider the following chain of weight reductions where $\mathcal{D}^{\prime}$ is a weight reduction of $\mathcal{D}$ on $x_{2}, \mathcal{D}^{\prime \prime}$ is a weight reduction of $\mathcal{D}^{\prime}$ on $x_{3}$, and $\mathcal{D}^{\prime \prime \prime}$ is a weight reduction of $\mathcal{D}^{\prime \prime}$ on $x_{2}$ with the corresponding edge ideals given as

$$
\begin{aligned}
I\left(\mathcal{D}^{\prime}\right) & =\left(x_{2} x_{1}, x_{3} x_{2}^{2}, x_{4} x_{3}^{2}, x_{4} x_{5}\right) \\
I\left(\mathcal{D}^{\prime \prime}\right) & =\left(x_{2} x_{1}, x_{3} x_{2}^{2}, x_{4} x_{3}, x_{4} x_{5}\right) \\
I\left(\mathcal{D}^{\prime \prime \prime}\right) & =\left(x_{2} x_{1}, x_{3} x_{2}, x_{4} x_{3}, x_{4} x_{5}\right) .
\end{aligned}
$$

\begin{tabular}{|c|c|c|c|c|c|}
\hline 0 & 1 & 2 & 3 & 4 & \\
\hline 0: & 1 & - & - & - & - \\
\hline 1: & - & 2 & - & - & - \\
\hline 2: & - & 1 & 2 & - & - \\
\hline 3: & - & 1 & 2 & 1 & - \\
\hline 4: & - & - & 2 & 3 & 1 \\
\hline
\end{tabular}

Below, we present the Betti tables of $I(\mathcal{D}), I\left(\mathcal{D}^{\prime}\right), I\left(\mathcal{D}^{\prime \prime}\right)$, and $I\left(\mathcal{D}^{\prime \prime \prime}\right)$, in order.

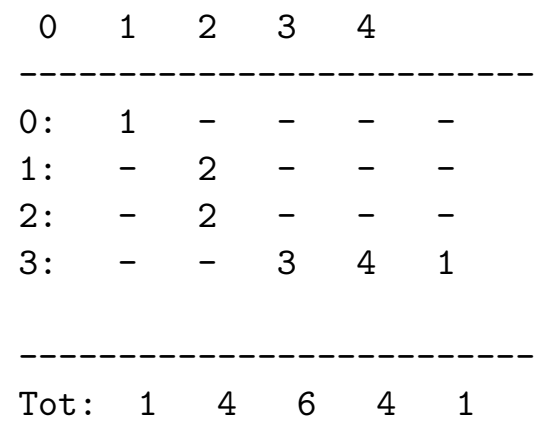

$\begin{array}{lcccc} & 0 & 1 & 2 & 3 \\ - & & 3 \\ 0: & 1 & - & - & - \\ 1: & - & 3 & 1 & - \\ 2: & - & 1 & 4 & 2\end{array}$

Tot: $1 \quad 4 \quad 5 \quad 2$

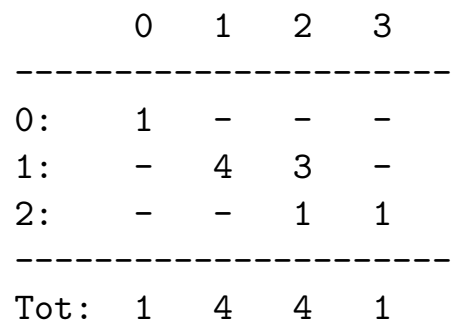


Based on the above Betti tables, equalities in Question 38 (a),(c),(d) hold for $\mathcal{D}$ and $\mathcal{D}^{\prime}$. However, we have

$$
\begin{gathered}
\operatorname{pdim}\left(R / I\left(\mathcal{D}^{\prime}\right)\right)=\operatorname{pdim}\left(R / I\left(\mathcal{D}^{\prime \prime}\right)\right)+1, \\
\operatorname{reg}\left(R / I\left(\mathcal{D}^{\prime \prime}\right)\right)=\operatorname{reg}\left(R / I\left(\mathcal{D}^{\prime \prime \prime}\right)\right),
\end{gathered}
$$

indicating that suggested equalities in Question 38 are not always valid. Computational experiments suggest that the desired equalities hold for $\mathcal{D}$ and a weight reduction of $\mathcal{D}$ on $x_{i}$ where $w_{i}>2$.

Answering above questions can help towards improving our understanding on the behavior of Betti numbers of monomial ideals under certain monomial operations.

\section{Acknowledgements}

The authors thank an anonymous referee for their valuable feedback and recommending the localization approach which lead to Observation 37. Many of the computations related to this project was done using Macaulay2 [13].

\section{References}

[1] Guillermo Alesandroni. Monomial ideals with large projective dimension. J. Pure Appl. Algebra, 224(6):106257, 13, 2020.

[2] Arindam Banerjee, Selvi Kara Beyarslan, and Hà Huy Tài. Regularity of edge ideals and their powers. In Southern Regional Algebra Conference, pages 17-52. Springer, 2017.

[3] Dave Bayer, Hara Charalambous, and Sorin Popescu. Extremal Betti numbers and applications to monomial ideals. J. Algebra, 221(2):497-512, 1999.

[4] Selvi Beyarslan, Huy Tài Hà, and Trân Nam Trung. Regularity of powers of forests and cycles. J. Algebraic Combin., 42(4):1077-1095, 2015.

[5] Selvi Kara Beyarslan, Jennifer Biermann, Kuei-Nuan Lin, and Augustine O'Keefe. Algebraic invariants of weighted oriented graphs. arXiv:1910.11773, 2019.

[6] Rachelle R. Bouchat. Free resolutions of some edge ideals of simple graphs. J. Commut. Algebra, 2(1):1-35, 2010.

[7] Rachelle R. Bouchat, Huy Tài Hà, and Augustine O'Keefe. Path ideals of rooted trees and their graded Betti numbers. J. Combin. Theory Ser. A, 118(8):2411-2425, 2011.

[8] Cícero Carvalho, Victor G. L. Neumann, and Hiram H. López. Projective nested cartesian codes. Bull. Braz. Math. Soc. (N.S.), 48(2):283-302, 2017.

[9] Shalom Eliahou and Michel Kervaire. Minimal resolutions of some monomial ideals. $J$. Algebra, 129(1):1-25, 1990.

[10] Christopher A. Francisco, Huy Tài Hà, and Adam Van Tuyl. Splittings of monomial ideals. Proc. Amer. Math. Soc., 137(10):3271-3282, 2009.

[11] Christopher A. Francisco, Jeffrey Mermin, and Jay Schweig. A survey of Stanley-Reisner theory. In Connections between algebra, combinatorics, and geometry, volume 76 of Springer Proc. Math. Stat., pages 209-234. Springer, New York, 2014.

[12] Philippe Gimenez, José Martínez-Bernal, Aron Simis, Rafael H. Villarreal, and Carlos E. Vivares. Symbolic powers of monomial ideals and Cohen-Macaulay vertex-weighted digraphs. In Singularities, algebraic geometry, commutative algebra, and related topics, pages 491-510. Springer, Cham, 2018. 
[13] Daniel R. Grayson and Michael E. Stillman. Macaulay2, a software system for research in algebraic geometry. Available at http://www.math.uiuc.edu/Macaulay2/.

[14] Huy Tài Hà, Kuei-Nuan Lin, Susan Morey, Enrique Reyes, and Rafael H. Villarreal. Edge ideals of oriented graphs. Internat. J. Algebra Comput., 29(3):535-559, 2019.

[15] Sean Jacques. Betti numbers of graph ideals. Ph.D. Thesis, The University of Sheffield, arXiv:math/0410107, 2004.

[16] José Martínez-Bernal, Yuriko Pitones, and Rafael H. Villarreal. Minimum distance functions of graded ideals and Reed-Muller-type codes. J. Pure Appl. Algebra, 221(2):251-275, 2017.

[17] Irena Peeva. Graded syzygies, volume 14 of Algebra and Applications. Springer-Verlag London, Ltd., London, 2011.

[18] Yuriko Pitones, Enrique Reyes, and Jonathan Toledo. Monomial ideals of weighted oriented graphs. Electron. J. Combin., 26(3): \#P3.44, 18, 2019.

[19] Guangjun Zhu, Li Xu, Hong Wang, Zhongming Tang, et al. Projective dimension and regularity of edge ideals of some weighted oriented graphs. Rocky Mountain Journal of Mathematics, 49(4):1391-1406, 2019. 